CRYSTALLOGRAPHIC COMMUNICATIONS

ISSN 2056-9890

Received 2 January 2016

Accepted 18 January 2016

Edited by M. Gdaniec, Adam Mickiewicz University, Poland

Keywords: crystal structure; dimethyl; diethyl; dicarboxylic acid; pyrazine; pyridine; $\mathrm{C}-\mathrm{H}$... and $\mathrm{C}-\mathrm{H} \ldots \mathrm{N}$ hydrogen bonding

CCDC references: $1448182 ; 1448181$ Supporting information: this article has supporting information at journals.iucr.org/e

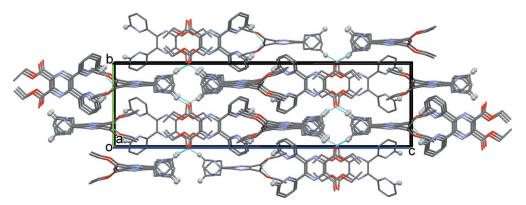

OPEN $\odot$ ACCESS

\section{Dimethyl and diethyl esters of 5,6-bis(pyridin-2-yl)- pyrazine-2,3-dicarboxylic acid: a comparison}

\author{
Montserrat Alfonso $^{\mathrm{a}}$ and Helen Stoeckli-Evans ${ }^{\mathrm{b} *}$ \\ ${ }^{\mathbf{a}}$ Institute of Chemistry, University of Neuchâtel, Av. de Bellevaux 51, CH-2000 Neuchâtel, Switzerland, and ${ }^{\mathbf{b}}$ Insitute of \\ Physics, University of Neuchâtel, rue Emile-Argand 11, CH-2000 Neuchâtel, Switzerland. *Correspondence e-mail: \\ helen.stoeckli-evans@unine.ch
}

In dimethyl 5,6-bis(pyridin-2-yl)pyrazine-2,3-dicarboxylate, $\mathrm{C}_{18} \mathrm{H}_{14} \mathrm{~N}_{4} \mathrm{O}_{4}$, (I), and diethyl 5,6-bis(pyridin-2-yl)pyrazine-2,3-dicarboxylate, $\mathrm{C}_{20} \mathrm{H}_{18} \mathrm{~N}_{4} \mathrm{O}_{4}$, (II), the dimethyl and diethyl esters of 5,6-bis(pyridin-2-yl)pyrazine-2,3-dicarboxylic acid, the orientation of the two pyridine rings differ. In (I), pyridine $\operatorname{ring} B$ is inclined to pyrazine ring $A$ by $44.8(2)^{\circ}$ and the pyridine and pyrazine $\mathrm{N}$ atoms are trans to one another, while pyridine ring $C$ is inclined to the pyrazine ring by $50.3(2)^{\circ}$, with the pyridine and pyrazine $\mathrm{N}$ atoms cis to one another. In compound (II), the diethyl ester, which possesses twofold rotation symmetry, the pyridine ring is inclined to the pyrazine ring by $40.7(1)^{\circ}$, with the pyridine and pyrazine $\mathrm{N}$ atoms trans to one another. In the crystal of (I), molecules are linked by $\mathrm{C}-\mathrm{H} \cdots \mathrm{N}$ hydrogen bonds, forming chains along [001]. The chains are linked by $\mathrm{C}-\mathrm{H} \cdots \pi$ interactions, forming a three-dimensional structure. In the crystal of (II), molecules are linked via $\mathrm{C}-\mathrm{H} \cdots \mathrm{O}$ hydrogen bonds, forming a threedimensional framework. There are $\mathrm{C}-\mathrm{H} \cdots \pi$ interactions present within the framework.

\section{Chemical context}

5,6-Bis(pyridin-2-yl)pyrazine-2,3-dicarboxylic acid ( $\left.\mathbf{L 1 H}_{2}\right)$ was synthesized to study its coordination behaviour with first row transitions metals (Alfonso, 1999). It exists as a zwitterion, with the adjacent pyridine and pyridinium rings almost coplanar due to the presence of an intramolecular $\mathrm{N}-\mathrm{H} \cdots \mathrm{N}$ hydrogen bond. The crystal structures of the zwitterion and different charged forms of $\mathbf{L 1 H}_{2}$, viz. the $\mathrm{HCl}, \mathrm{HClO}_{4}$ and $\mathrm{HPF}_{6}$ salts, and details of the hydrogen bonding have been reported (Alfonso et al., 2001).

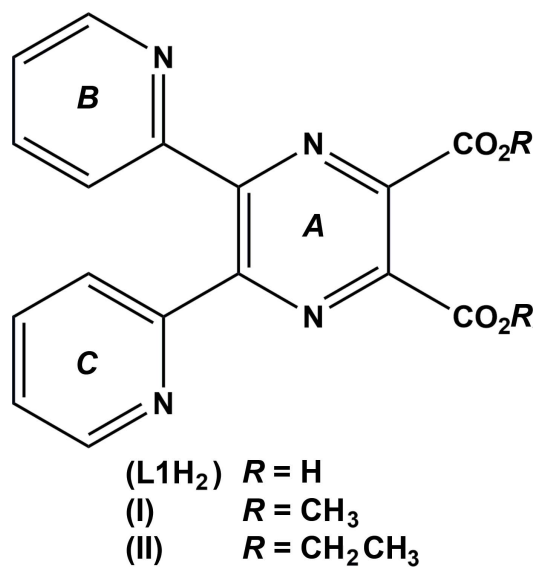

Metal-catalysed hydrolysis of amino acid esters is a well documented phenomenon (Dugas, 1989). It has been shown previously that the reaction of copper(II) salts with the dimethyl esters of pyrazine-2,3-dicarboxylic acid (Neels et al., 


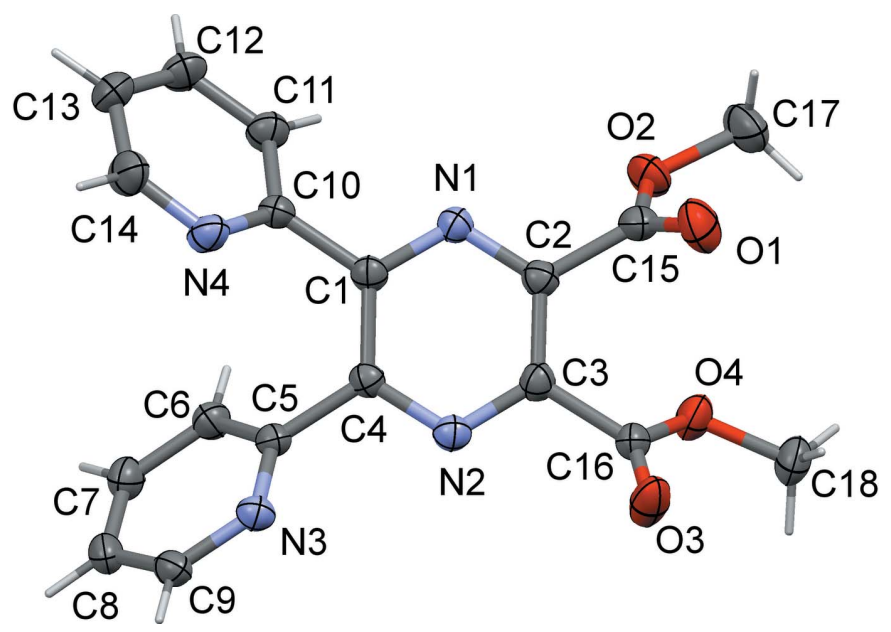

Figure 1

A view of the molecular structure of compound (I), showing the atom labelling. Displacement ellipsoids are drawn at the $50 \%$ probability level.

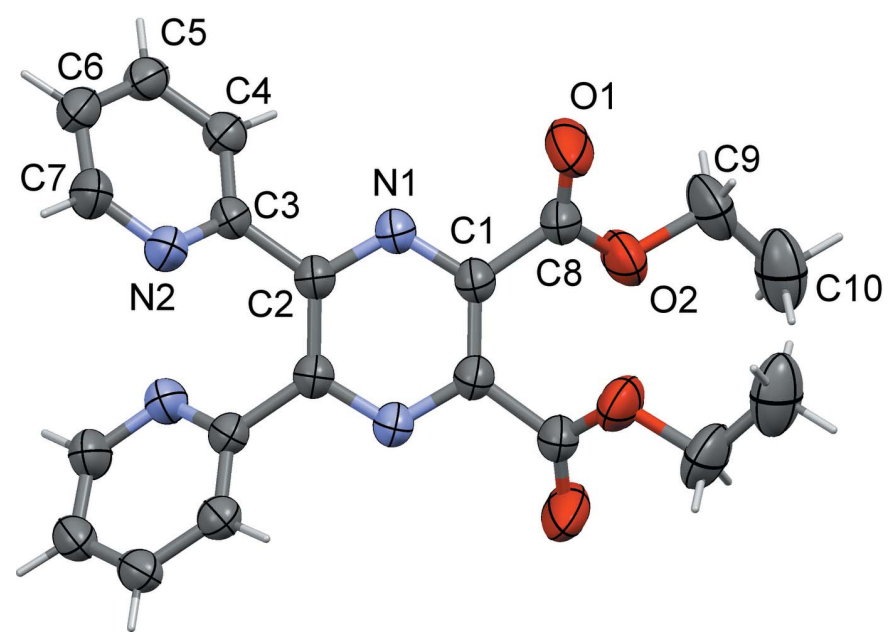

Figure 2

A view of the molecular structure of compound (II), showing the atom labelling. Displacement ellipsoids are drawn at the $50 \%$ probability level. Unlabelled atoms are related to labelled atoms by the symmetry code $\left(-x+2,-y+\frac{3}{2}, z\right)$.

1997) and 2,5-dimethylpyrazine-3,6-dicarboxylic acid (Wang \& Stoeckli-Evans, 1998) resulted in the partial hydrolysis of the ligand and the formation of a two-dimensional network in the first case and a mononuclear complex in the second. Hence, metal-ion-promoted ester hydrolysis leads to the formation of new ligands and may serve as a general route to prepare new coordination compounds. The title compounds, (I) and (II), were synthesized to study the hydrolysis of these esters with first row transition metals (Alfonso, 1999), and we report herein on their syntheses and crystal structures.

\section{Structural commentary}

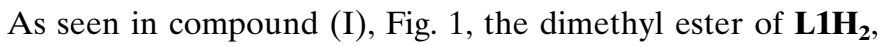
pyridine ring $B(\mathrm{~N} 4 / \mathrm{C} 10-\mathrm{C} 14)$ is inclined to the pyrazine ring
Table 1

Hydrogen-bond geometry $\left(\AA,^{\circ}\right)$ for (I).

$C g 2$ is the centroid of the N3/C5-C9 pyridine ring.

\begin{tabular}{lllll}
\hline$D-\mathrm{H} \cdots A$ & $D-\mathrm{H}$ & $\mathrm{H} \cdots A$ & $D \cdots A$ & $D-\mathrm{H} \cdots A$ \\
\hline $\mathrm{C} 11-\mathrm{H} 11 \cdots \mathrm{N} 3^{\mathrm{i}}$ & 0.93 & 2.57 & $3.334(5)$ & 140 \\
$\mathrm{C} 7-\mathrm{H} 7 \cdots C g 2^{\mathrm{ii}}$ & 0.93 & 2.95 & $3.742(5)$ & 144 \\
$\mathrm{C} 17-\mathrm{H} 17 C \cdots C g 2^{\mathrm{iii}}$ & 0.96 & 2.92 & $3.722(6)$ & 141 \\
\hline
\end{tabular}

Symmetry codes: (i) $x,-y+\frac{3}{2}, z-\frac{1}{2}$; (ii) $x+\frac{1}{2},-y+1, z$; (iii) $x-\frac{1}{2}, y+\frac{3}{2}, z-\frac{1}{2}$.

$(A ; \mathrm{N} 1 / \mathrm{N} 2 / \mathrm{C} 1-\mathrm{C} 4)$ by $44.8(2)^{\circ}$ and the pyridine and pyrazine $\mathrm{N}$ atoms, $\mathrm{N} 1$ and $\mathrm{N} 4$, are trans to one another. Pyridine ring $C$ (N3/C5-C9) is inclined to pyrazine ring $A$ by $50.3(2)^{\circ}$. However, here the pyridine and pyrazine $\mathrm{N}$ atoms, N2 and N3, are $c i s$ to one another. The two pyridine rings, $B$ and $C$, are inclined to one another by $60.2(2)^{\circ}$. The acetate groups, $\mathrm{O} 1 / \mathrm{O} 2 / \mathrm{C} 15 / \mathrm{C} 17$ and $\mathrm{O} 3 / \mathrm{O} 4 / \mathrm{C} 16 / \mathrm{C} 18$, are almost planar with r.m.s. deviations of 0.027 and $0.007 \AA$, respectively. They are inclined to the pyrazine ring by $60.3(3)$ and $49.8(3)^{\circ}$, respectively, and to one another by $42.4(3)^{\circ}$.

Compound (II), the diethyl ester of $\mathbf{L} \mathbf{1 H _ { 2 }}$, possesses twofold rotation symmetry, with the twofold rotation axis bisecting the $\mathrm{C}_{\mathrm{ar}}-\mathrm{C}_{\mathrm{ar}}^{\mathrm{i}}$ bonds [ar = aromatic; symmetry code (i): $-x+2$, $\left.-y+\frac{3}{2}, z\right]$, as shown in Fig. 2. The pyridine $\mathrm{N}$ atoms, $\mathrm{N} 2$ and $\mathrm{N} 2{ }^{\mathrm{i}}$, face one another with an $\mathrm{N} 2 \cdots \mathrm{N} 2^{\mathrm{i}}$ separation of 3.043 (3) $\AA$. The two pyridine rings are inclined to one another by $55.1(1)^{\circ}$ and to the pyrazine ring mean plane by $40.7(1)^{\circ}$, with the pyrazine and pyridine $\mathrm{N}$ atoms, $\mathrm{N} 1$ and $\mathrm{N} 2$, trans to one another. The acetate group, O1/O2/C8/C9 [maximum deviation of 0.012 (3) $\AA$ for atom C8] is inclined to the pyrazine ring mean plane by $38.9(1)^{\circ}$, and by $47.6(2)^{\circ}$ to the acetate group related by the twofold rotation axis. The oxygen atoms, $\mathrm{O} 2$ and $\mathrm{O} 2{ }^{\mathrm{i}}$, are separated by only 2.840 (3) $\AA$. The pyrazine ring in (II) has a slight twist-boat conformation (r.m.s. deviation $=0.046 \AA$ ) with the $\mathrm{N} 1 / \mathrm{C} 1 / \mathrm{C} 2$ and $\mathrm{N} 1{ }^{\mathrm{i}} / \mathrm{C} 1^{\mathrm{i}} / \mathrm{C} 2^{\mathrm{i}}$ planes inclined to one another by $3.9(3)^{\circ}$.

As noted above the differences in the structures of the two compounds lies essentially in the orientation of the pyridine rings with respect to the pyrazine ring ( $c f$ Figs. 1 and 2). It is possible that the slight distortion of the planarity of the pyrazine ring in (II), mentioned above, is related to the short $\mathrm{N} 2 \cdots \mathrm{N} 22^{\mathrm{i}}$ contact of 3.043 (3) $\AA$ of the adjacent pyridine rings and to the even shorter $\mathrm{O} 2 \cdots \mathrm{O} 22^{\mathrm{i}}$ contact of 2.840 (3) $\AA$ of the adjacent acetate groups.

\section{Supramolecular features}

In the crystal of (I), molecules are linked by $\mathrm{C}-\mathrm{H} \cdots \mathrm{N}$ hydrogen bonds, forming chains along [001]; see Table 1 and Fig. 3. The chains are linked via $\mathrm{C}-\mathrm{H} \cdots \pi$ interactions (Table 1), forming a three-dimensional structure.

In the crystal of (II), molecules are linked via $\mathrm{C}-\mathrm{H} \cdots \mathrm{O}$ hydrogen bonds, forming a three-dimensional framework; see Table 2 and Fig. 4. Within the framework there are a number of $\mathrm{C}-\mathrm{H} \cdots \pi$ interactions present (Table 2). 
Table 2

Hydrogen-bond geometry $\left(\AA,^{\circ}\right)$ for (II).

$C g 1$ and $C g 2$ are the centroids of the pyrazine and pyridine rings $\mathrm{N} 1 / \mathrm{C} 1 / \mathrm{C} 2 /$ $\mathrm{N} 1^{\prime} / \mathrm{Cl}^{\prime} / \mathrm{C}^{\prime}$ and $\mathrm{N} 2 / \mathrm{C} 3-\mathrm{C} 7$, respectively [symmetry code $\left.\left({ }^{\prime}\right):-x+2,-y+\frac{3}{2}, z\right]$.

\begin{tabular}{lllll}
\hline$D-\mathrm{H} \cdots A$ & $D-\mathrm{H}$ & $\mathrm{H} \cdots A$ & $D \cdots A$ & $D-\mathrm{H} \cdots A$ \\
\hline $\mathrm{C} 7-\mathrm{H} 7 \cdots \mathrm{O} 1^{\mathrm{i}}$ & 0.94 & 2.48 & $3.308(3)$ & 147 \\
$\mathrm{C} 4-\mathrm{H} 4 \cdots C g 2^{\mathrm{ii}}$ & 0.94 & 2.92 & $3.739(2)$ & 147 \\
$\mathrm{C} 10-\mathrm{H} 10 B \cdots C g 1^{\mathrm{iii}}$ & 0.97 & 2.56 & $3.409(3)$ & 146 \\
$\mathrm{C} 10-\mathrm{H} 10 B \cdots C g 1^{\text {iv }}$ & 0.97 & 2.56 & $3.409(3)$ & 146 \\
\hline
\end{tabular}

Symmetry codes: (i) $\quad-y+\frac{7}{4}, x+\frac{1}{4}, z+\frac{1}{4} ; \quad$ (ii) $y-\frac{1}{4},-x+\frac{5}{4},-z+\frac{1}{4} ; \quad$ (iii) $-x+2,-y+1,-z ;$ (iv) $x, y-\frac{1}{2},-z$.

\section{Database survey}

Besides the structures of the zwitterion and different charged forms of $\mathbf{L 1 H}_{2}, v i z$. the $\mathrm{HCl}, \mathrm{HClO}_{4}$ and $\mathrm{HPF}_{6}$ salts (Alfonso et al., 2001), the crystal structures of two copper(II) complexes of

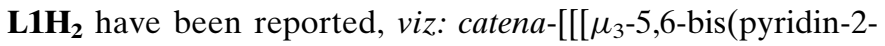
yl)pyrazine-2,3-dicarboxylate]triaquadibromodicopper(II)] methanol solvate trihydrate $]$ and catena- $\left[\left[\left[\mu_{4}-5,6\right.\right.\right.$-bis(pyridin2-yl)pyrazine-2,3-dicarboxylate)diaquadibromodicopper(II) monohydrate] (Neels et al., 2003).

The structure of the isoelectronic compound 3,6-bis(pyridin-2-yl)pyrazine-2,5-dicarboxylic acid $\left(\mathbf{L 2} \mathbf{H}_{2}\right)$, Fig. 5, has also been reported (Wang \& Stoeckli-Evans, 2012a). It too exists as a zwitterion and the structures of its dihydrochloride salt and the dimethyl sulfonate disolvate have also been reported (Wang \& Stoeckli-Evans, 2012a). The crystal structures of the dimethyl (III) and diethyl (IV) esters of $\mathbf{2} 2 \mathbf{H}_{2}$ have been deposited as private communications (Wang \& Stoeckli-Evans, 2012b,c) with the Cambridge Structural Database (CSD; Groom \& Allen, 2014). Both compounds crystallize in the triclinic space group $P \overline{1}$ and possess inversion symmetry. The pyridine rings lie almost in the plane of the pyrazine ring and the $\mathrm{N}$ atoms are trans with respect to each other and to the nearest pyrazine $\mathrm{N}$ atom (as illustrated in Fig. 5). The ester groups are planar and in both compounds lie almost normal to the pyrazine ring. In the crystals of both compounds, inversion-related molecules are linked via pairs of $\mathrm{C}-\mathrm{H} \cdots \mathrm{O}$ hydrogen bonds, enclosing $R_{2}^{2}(10)$ ring motifs,

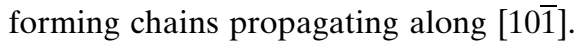

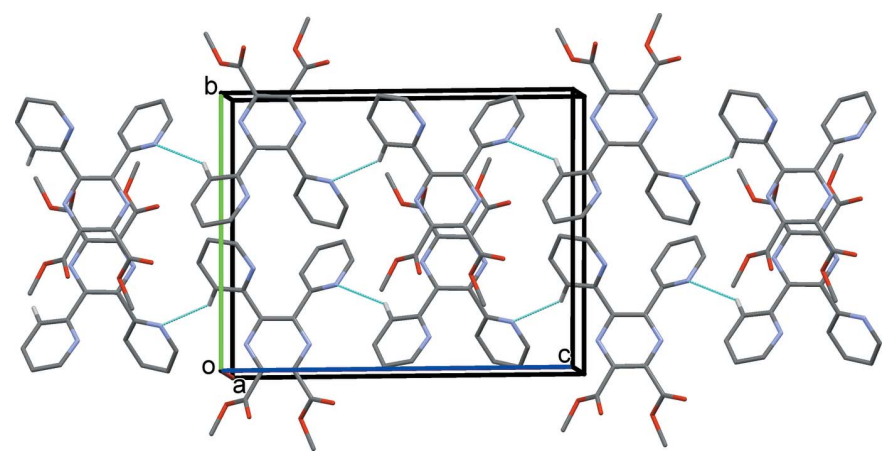

Figure 3

A view along the $a$ axis of the crystal packing of compound (I). The hydrogen bonds are shown as dashed lines (see Table 1; only H atom H11 has been included).

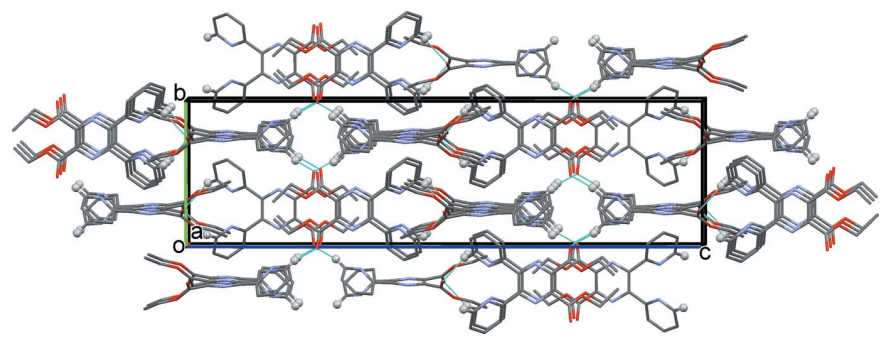

Figure 4

A view along the $a$ axis of the crystal packing of compound (II). The hydrogen bonds are shown as dashed lines (see Table 2; only $\mathrm{H}$ atom $\mathrm{H} 7$ has been included).

\section{Synthesis and crystallization}

The synthesis of 5,6-bis(pyridin-2-yl)pyrazine-2,3-dicarboxylic acid $\left(\mathbf{L} 1 \mathbf{H}_{2}\right)$ has been reported (Alfonso et al., 2001). The dimethyl and diethyl esters, compounds (I) and (II), respectively, were obtained by the usual esterification procedure in acidic medium from the diacid and an excess of the corresponding alcohol.

Synthesis of compound (I): dimethyl-5,6-bis(pyridin-2-yl)pyrazine-2,3-dicarboxylate $\mathrm{L1H}_{2}$

$(1.00 \mathrm{~g}, 3.11 \mathrm{mmol})$ was heated under reflux in freshly distilled $\mathrm{MeOH}(40 \mathrm{ml})$ containing $\mathrm{H}_{2} \mathrm{SO}_{4}$ conc. $(98 \%, 1 \mathrm{ml})$ during $16 \mathrm{~h}$. After stopping the reaction, the temperature of the solution was allowed to cool to room temperature and then poured into an aqueous solution of $\mathrm{NaOAc}(6 \mathrm{~g}$ in $150 \mathrm{ml}$ deionized water). The resulting solution was stirred in an ice bath containing $\mathrm{NaCl}$ to afford a white solid which was removed by filtration, washed with cold water and dried under vacuum. Single crystals suitable for $\mathrm{X}$-ray analysis were obtained by the slow diffusion technique from $\mathrm{CH}_{2} \mathrm{Cl}_{2}$ and $\mathrm{MeOH}$ (yield: $0.77 \mathrm{~g}, 65 \%$; m.p. 410.2-411.7 K). Selected IR bands $\left(\mathrm{KBr}\right.$ pellet, $\left.\mathrm{cm}^{-1}\right): v=1743(s), 1729(v s), 1339(s)$, 1302(s), 1283(vs), 1164(s), 1089(vs). ${ }^{1} \mathrm{H}$ NMR (CDCl3, $400 \mathrm{MHz}$, p.p.m.): $\delta=8.34(d t, 2 \mathrm{H}, J=4.1 \mathrm{~Hz}, J=1.0 \mathrm{~Hz}, \mathrm{pyH})$,

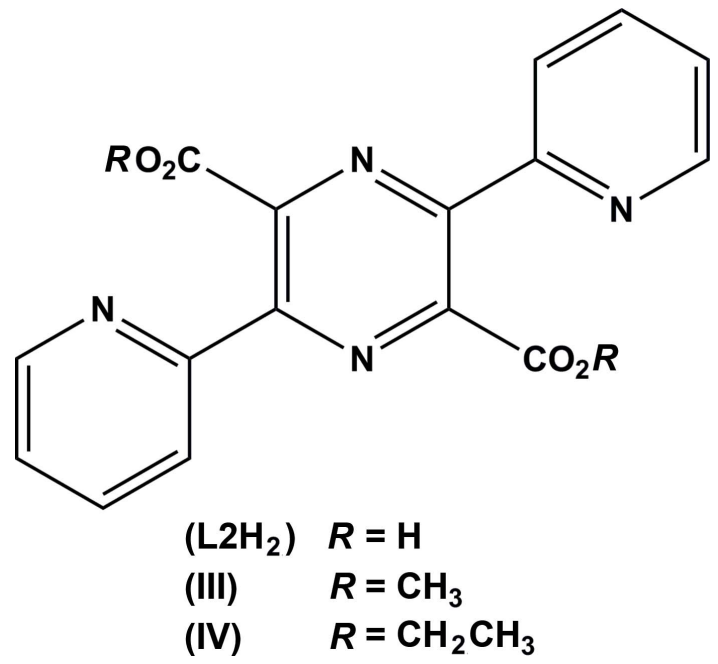

Figure 5

The chemical scheme for compound $\mathbf{L} \mathbf{2} \mathbf{H}_{2}$. 
Table 3

Experimental details.

(I)

Crystal data

$M_{\mathrm{r}}$

Crystal system, space group

Temperature (K)

$a, b, c(\AA)$

$\alpha, \beta, \gamma\left({ }^{\circ}\right)$

$V\left(\AA^{3}\right)$

$Z$

Radiation type

$\mu\left(\mathrm{mm}^{-1}\right)$

Crystal size $(\mathrm{mm})$

Data collection

Diffractometer

No. of measured, independent and observed

$[I>2 \sigma(I)]$ reflections

$R_{\text {int }}$

$(\sin \theta / \lambda)_{\max }\left(\AA^{-1}\right)$

Refinement

$R\left[F^{2}>2 \sigma\left(F^{2}\right)\right], w R\left(F^{2}\right), S$

No. of parameters

No. of restraints

$\mathrm{H}$-atom treatment
Chemical formula

No. of reflections

$\Delta \rho_{\max }, \Delta \rho_{\min }\left(\mathrm{e} \AA^{-3}\right)$

\author{
$\mathrm{C}_{18} \mathrm{H}_{14} \mathrm{~N}_{4} \mathrm{O}_{4}$ \\ 350.33 \\ Monoclinic, Ia \\ 293 \\ 8.4249 (12), 12.2465 (10), 16.2561 (13) \\ 90, $103.730(8), 90$ \\ $1629.3(3)$ \\ 4 \\ Mo $K \alpha$ \\ 0.10 \\ $0.70 \times 0.50 \times 0.38$
}

(II)

Computer programs: STADI4 (Stoe \& Cie, 1997), EXPOSE, CELL and INTEGRATE in IPDS-I (Stoe \& Cie, 2004), X-RED (Stoe \& Cie, 1997), SHELXS97 (Sheldrick, 2008), SHELXL2014 (Sheldrick, 2015), PLATON (Spek, 2009), Mercury (Macrae et al., 2008), and publCIF (Westrip, 2010). 7.99(dt, 2H, $J=7.7 \mathrm{~Hz}, J=1.0 \mathrm{~Hz}, \mathrm{pyH}), 7.82(t d, 2 \mathrm{H}, J=$ $7.7 \mathrm{~Hz}, J=1.0 \mathrm{~Hz}, \mathrm{pyH}), 7.26(t d, 2 \mathrm{H}, J=7.7 \mathrm{~Hz}, J=1.0 \mathrm{~Hz}$, pyH), $4.04\left(s, 6 \mathrm{H}, \mathrm{CH}_{3}\right) \cdot{ }^{13} \mathrm{C}$ NMR $\left(\mathrm{CDCl}_{3}, 50 \mathrm{MHz}\right.$, p.p.m.): $\delta$ $=165.53,155.98,153.35,149.26,142.92,137.64,125.41,124.49$, 54.11. DCI-MS $m / z: 351\left(M^{+}\right), 318,279,255,208$. Analysis for $\mathrm{C}_{18} \mathrm{H}_{14} \mathrm{~N}_{4} \mathrm{O}_{4}$ (350.33), calculated $\mathrm{C} 61.70, \mathrm{H} 4.04, \mathrm{~N}$ $15.99 \%$, found C $61.4, \mathrm{H} 3.91$, N $15.65 \%$.

\section{Synthesis of compound (II): diethyl-5,6-bis(pyridin-2-yl)- pyrazine-2,3-dicarboxylate}

This compound was prepared by the same method as for (I). $\mathbf{L 1 H}_{\mathbf{2}}$ in freshly distilled EtOH containing catalytic amounts of $\mathrm{H}_{2} \mathrm{SO}_{4}$ conc. gave compound (II) as a white solid. Slow evaporation of an ethanolic solution afforded colourless crystals suitable for X-ray analysis (yield: $0.70 \mathrm{~g}, 62 \%$; m.p. 390.5-391.3 K). Selected IR bands (KBr pellet, $\left.\mathrm{cm}^{-1}\right): v=$ $3055(w), \quad 1737(s), \quad 1723(v s), \quad 1368(s), \quad 1301(s), \quad 1276(v s)$, 1276(vs), 1161(s), 1086(vs). ${ }^{1} \mathrm{H}$ NMR $\left(\mathrm{CDCl}_{3}, 400 \mathrm{MHz}\right.$, p.p.m.): $\delta=8.33(d, 2 \mathrm{H}, J=4 \mathrm{~Hz}, \mathrm{pyH}), 8.01(d, 2 \mathrm{H}, J=7.7 \mathrm{~Hz}$, pyH), $7.81(t, 2 \mathrm{H}, J=7.7 \mathrm{~Hz}$, pyH $), 7.24(t, 2 \mathrm{H}, J=4.4 \mathrm{~Hz}$, pyH$)$, $4.52\left(m, 4 \mathrm{H}, J=7 \mathrm{~Hz}, \mathrm{CH}_{2}\right), 1.45\left(t, 6 \mathrm{H}, J=7.4 \mathrm{~Hz}, \mathrm{CH}_{3}\right)$. EIMS $m / z: 378(34), 349$ (9), 232 (95), 206 (66), $179(25)$, 152 (11), 129 (9), 78(base), 46 (38). Analysis for $\mathrm{C}_{20} \mathrm{H}_{18} \mathrm{~N}_{4} \mathrm{O}_{4}$ (378.38), calculated C 63.49, H 4.79, N $14.81 \%$, found C 63.49, H 4.61, N $14.77 \%$.

\section{Refinement}

Crystal data, data collection and structure refinement details are summarized in Table 3. For both compounds, the C-bound
$\mathrm{H}$-atoms were included in calculated positions and treated as riding atoms: $\mathrm{C}-\mathrm{H}=0.93-0.98 \AA$ with $U_{\text {iso }}(\mathrm{H})=1.5 U_{\text {eq }}(\mathrm{C}$ methyl) and $1.2 U_{\text {eq }}(\mathrm{C})$ for other $\mathrm{H}$ atoms. For compound (I), the Flack parameter (Parsons et al., 2013) is $=-0.2$ (10), but it has no physical meaning here.

\section{Acknowledgements}

We are grateful to the Swiss National Science Foundation and the University of Neuchâtel for financial support.

\section{References}

Alfonso, M. (1999). PhD thesis, University of Neuchâtel, Switzerland. Alfonso, M., Wang, Y. \& Stoeckli-Evans, H. (2001). Acta Cryst. C57, 1184-1188.

Dugas, H. (1989). In Bioorganic Chemistry: A Chemical Approach to Enzyme Action. 2nd ed. Berlin: Springer-Verlag.

Groom, C. R. \& Allen, F. H. (2014). Angew. Chem. Int. Ed. 53, 662671.

Macrae, C. F., Bruno, I. J., Chisholm, J. A., Edgington, P. R., McCabe, P., Pidcock, E., Rodriguez-Monge, L., Taylor, R., van de Streek, J. \& Wood, P. A. (2008). J. Appl. Cryst. 41, 466-470.

Neels, A., Alfonso, M., Mantero, D. G. \& Stoeckli-Evans, H. (2003). Chimia, 57, 619-622.

Neels, A., Stoeckli-Evans, H., Wang, Y., Clearfield, A. \& Poojary, D. M. (1997). Inorg. Chem. 36, 5406-5408.

Parsons, S., Flack, H. D. \& Wagner, T. (2013). Acta Cryst. B69, 249259.

Sheldrick, G. M. (2008). Acta Cryst. A64, 112-122.

Sheldrick, G. M. (2015). Acta Cryst. C71, 3-8.

Spek, A. L. (2009). Acta Cryst. D65, 148-155. 
Stoe \& Cie (1997). STADI4 and X-RED. Stoe \& Cie GmbH, Damstadt, Germany.

Stoe \& Cie (2004). IPDS-I Bedienungshandbuch. Stoe \& Cie GmbH, Darmstadt, Germany.

Wang, Y. \& Stoeckli-Evans, H. (1998). Acta Cryst. C54, 306-308.

Wang, Y. \& Stoeckli-Evans, H. (2012a). Acta Cryst. C68, o431-o435.
Wang, Y. \& Stoeckli-Evans, H. (2012b). Private communication (refcode 914647). CCDC, Cambridge, England.

Wang, Y. \& Stoeckli-Evans, H. (2012c). Private communication (refcode 914648). CCDC, Cambridge, England.

Westrip, S. P. (2010). J. Appl. Cryst. 43, 920-925. 


\section{supporting information}

Acta Cryst. (2016). E72, 233-237 [doi:10.1107/S2056989016001080]

\section{Dimethyl and diethyl esters of 5,6-bis(pyridin-2-yl) pyrazine-2,3-dicarboxylic} acid: a comparison

\section{Montserrat Alfonso and Helen Stoeckli-Evans}

\section{Computing details}

Data collection: STADI4 (Stoe \& Cie, 1997) for (I); EXPOSE in IPDS-I (Stoe \& Cie, 2004) for (II). Cell refinement: STADI4 (Stoe \& Cie, 1997) for (I); CELL in IPDS-I (Stoe \& Cie, 2004) for (II). Data reduction: X-RED (Stoe \& Cie, 1997) for (I); INTEGRATE in IPDS-I (Stoe \& Cie, 2004) for (II). For both compounds, program(s) used to solve structure: SHELXS97 (Sheldrick, 2008); program(s) used to refine structure: SHELXL2014 (Sheldrick, 2015); molecular graphics: PLATON (Spek, 2009) and Mercury (Macrae et al., 2008); software used to prepare material for publication: SHELXL2014 (Sheldrick, 2015), PLATON (Spek, 2009) and publCIF (Westrip, 2010).

(I) Dimethyl 5,6-bis(pyridin-2-yl)pyrazine-2,3-dicarboxylate

Crystal data

$\mathrm{C}_{18} \mathrm{H}_{14} \mathrm{~N}_{4} \mathrm{O}_{4}$

$M_{r}=350.33$

Monoclinic, $I a$

$a=8.4249(12) \AA$

$b=12.2465(10) \AA$

$c=16.2561(13) \AA$

$\beta=103.730(8)^{\circ}$

$V=1629.3(3) \AA^{3}$

$Z=4$

\section{Data collection}

Stoe-Siemens AED2 diffractometer

$\omega / 2 \mathrm{q}$ scans

3035 measured reflections 3028 independent reflections 2737 reflections with $I>2 \sigma(I)$

$R_{\text {int }}=0.012$

\section{Refinement}

Refinement on $F^{2}$

Least-squares matrix: full

$R\left[F^{2}>2 \sigma\left(F^{2}\right)\right]=0.050$

$w R\left(F^{2}\right)=0.135$

$S=1.11$

3028 reflections

238 parameters

2 restraints
$F(000)=728$

$D_{\mathrm{x}}=1.428 \mathrm{Mg} \mathrm{m}^{-3}$

Mo $K \alpha$ radiation, $\lambda=0.71073 \AA$

Cell parameters from 33 reflections

$\theta=14.1-19.6^{\circ}$

$\mu=0.10 \mathrm{~mm}^{-1}$

$T=293 \mathrm{~K}$

Rod, colourless

$0.70 \times 0.50 \times 0.38 \mathrm{~mm}$

$\theta_{\max }=25.5^{\circ}, \theta_{\min }=2.1^{\circ}$

$h=-10 \rightarrow 10$

$k=0 \rightarrow 14$

$l=-19 \rightarrow 19$

2 standard reflections every $60 \mathrm{~min}$ intensity decay: $1 \%$

Primary atom site location: structure-invariant direct methods

Secondary atom site location: difference Fourier map

Hydrogen site location: inferred from neighbouring sites

$\mathrm{H}$-atom parameters constrained 
$w=1 /\left[\sigma^{2}\left(F_{\mathrm{o}}^{2}\right)+(0.0759 P)^{2}+1.0624 P\right]$

where $P=\left(F_{\mathrm{o}}^{2}+2 F_{\mathrm{c}}^{2}\right) / 3$

$(\Delta / \sigma)_{\max }<0.001$

$\Delta \rho_{\max }=0.19$ e $\AA^{-3}$
$\Delta \rho_{\min }=-0.21$ e $\AA^{-3}$

Extinction correction: SHELXL2014 (Sheldrick, 2015), $\mathrm{Fc}^{*}=\mathrm{kFc}\left[1+0.001 \mathrm{xFc}^{2} \lambda^{3} / \sin (2 \theta)\right]^{-1 / 4}$

Extinction coefficient: 0.0065 (18)

\section{Special details}

Geometry. All e.s.d.'s (except the e.s.d. in the dihedral angle between two l.s. planes) are estimated using the full covariance matrix. The cell e.s.d.'s are taken into account individually in the estimation of e.s.d.'s in distances, angles and torsion angles; correlations between e.s.d.'s in cell parameters are only used when they are defined by crystal symmetry. An approximate (isotropic) treatment of cell e.s.d.'s is used for estimating e.s.d.'s involving l.s. planes.

Fractional atomic coordinates and isotropic or equivalent isotropic displacement parameters $\left(\AA^{2}\right)$

\begin{tabular}{|c|c|c|c|c|}
\hline & $x$ & $y$ & $z$ & $U_{\mathrm{iso}} * / U_{\mathrm{eq}}$ \\
\hline $\mathrm{O} 1$ & $0.8419(5)$ & $1.1583(3)$ & $0.0691(2)$ & $0.0470(9)$ \\
\hline $\mathrm{O} 2$ & $1.0295(4)$ & $1.1079(3)$ & $-0.0014(2)$ & $0.0395(8)$ \\
\hline $\mathrm{O} 3$ & $1.0067(5)$ & $1.1074(3)$ & $0.3016(2)$ & $0.0463(10)$ \\
\hline $\mathrm{O} 4$ & $1.1454(4)$ & $1.1588(3)$ & $0.2054(2)$ & $0.0355(8)$ \\
\hline N1 & $0.9519(4)$ & $0.8948(3)$ & $0.0567(2)$ & $0.0266(8)$ \\
\hline $\mathrm{N} 2$ & $1.0688(4)$ & $0.8934(3)$ & $0.2330(2)$ & $0.0272(8)$ \\
\hline N3 & $1.0738(4)$ & $0.6793(3)$ & $0.3087(2)$ & $0.0279(8)$ \\
\hline N4 & $0.8579(5)$ & $0.6232(3)$ & $0.0803(2)$ & $0.0348(9)$ \\
\hline $\mathrm{C} 1$ & $0.9822(5)$ & 0.8015 (3) & $0.0992(2)$ & $0.0248(9)$ \\
\hline $\mathrm{C} 2$ & $0.9784(5)$ & $0.9878(3)$ & $0.1014(2)$ & $0.0259(9)$ \\
\hline $\mathrm{C} 3$ & $1.0319(5)$ & $0.9857(3)$ & $0.1896(3)$ & $0.0268(9)$ \\
\hline $\mathrm{C} 4$ & $1.0489(5)$ & $0.8006(3)$ & $0.1880(2)$ & $0.0227(8)$ \\
\hline $\mathrm{C} 5$ & $1.1158(5)$ & $0.6996(3)$ & $0.2355(2)$ & $0.0248(9)$ \\
\hline C6 & $1.2218(5)$ & 0.6347 (4) & $0.2045(3)$ & $0.0311(9)$ \\
\hline H6 & 1.2486 & 0.6520 & 0.1537 & $0.037 *$ \\
\hline $\mathrm{C} 7$ & $1.2879(6)$ & $0.5430(4)$ & $0.2500(3)$ & $0.0349(10)$ \\
\hline $\mathrm{H} 7$ & 1.3595 & 0.4979 & 0.2303 & $0.042 *$ \\
\hline $\mathrm{C} 8$ & $1.2459(6)$ & $0.5200(3)$ & $0.3248(3)$ & $0.0345(10)$ \\
\hline H8 & 1.2879 & 0.4589 & 0.3565 & $0.041^{*}$ \\
\hline $\mathrm{C} 9$ & $1.1394(5)$ & $0.5903(4)$ & $0.3517(3)$ & $0.0312(9)$ \\
\hline H9 & 1.1118 & 0.5748 & 0.4025 & $0.037 *$ \\
\hline $\mathrm{C} 10$ & $0.9393(5)$ & $0.6998(3)$ & $0.0486(2)$ & $0.0255(9)$ \\
\hline C11 & $0.9763(6)$ & 0.6908 (4) & $-0.0299(3)$ & $0.0334(10)$ \\
\hline H11 & 1.0322 & 0.7464 & -0.0499 & $0.040^{*}$ \\
\hline $\mathrm{C} 12$ & $0.9291(6)$ & 0.5984 (4) & $-0.0777(3)$ & 0.0389 (11) \\
\hline H12 & 0.9543 & 0.5900 & -0.1301 & $0.047^{*}$ \\
\hline $\mathrm{C} 13$ & $0.8443(7)$ & $0.5192(4)$ & $-0.0469(3)$ & $0.0413(12)$ \\
\hline H13 & 0.8098 & 0.4561 & -0.0779 & $0.050^{*}$ \\
\hline $\mathrm{C} 14$ & $0.8113(7)$ & $0.5353(4)$ & $0.0320(3)$ & 0.0439 (13) \\
\hline H14 & 0.7529 & 0.4814 & 0.0524 & $0.053^{*}$ \\
\hline $\mathrm{C} 15$ & $0.9399(6)$ & $1.0942(4)$ & $0.0546(3)$ & $0.0316(10)$ \\
\hline $\mathrm{C} 16$ & $1.0580(5)$ & $1.0903(4)$ & $0.2399(3)$ & $0.0287(9)$ \\
\hline $\mathrm{C} 17$ & $1.0131(8)$ & $1.2133(4)$ & $-0.0439(3)$ & $0.0525(14)$ \\
\hline H17A & 1.0924 & 1.2190 & -0.0774 & $0.079^{*}$ \\
\hline H17B & 1.0307 & 1.2706 & -0.0024 & $0.079 *$ \\
\hline
\end{tabular}




\begin{tabular}{lllll}
$\mathrm{H} 17 \mathrm{C}$ & 0.9053 & 1.2199 & -0.0798 & $0.079^{*}$ \\
$\mathrm{C} 18$ & $1.1672(6)$ & $1.2674(4)$ & $0.2412(3)$ & $0.0386(11)$ \\
$\mathrm{H} 18 \mathrm{~A}$ & 1.2114 & 1.3143 & 0.2049 & $0.058^{*}$ \\
$\mathrm{H} 18 \mathrm{~B}$ & 1.2410 & 1.2645 & 0.2961 & $0.058^{*}$ \\
$\mathrm{H} 18 \mathrm{C}$ & 1.0636 & 1.2955 & 0.2464 & $0.058^{*}$ \\
\hline
\end{tabular}

Atomic displacement parameters $\left(\AA^{2}\right)$

\begin{tabular}{lllllll}
\hline & $U^{11}$ & $U^{22}$ & $U^{33}$ & $U^{12}$ & $U^{13}$ & $U^{23}$ \\
\hline O1 & $0.054(2)$ & $0.0354(19)$ & $0.054(2)$ & $0.0149(17)$ & $0.0183(17)$ & $0.0094(17)$ \\
O2 & $0.055(2)$ & $0.0344(19)$ & $0.0313(17)$ & $-0.0011(15)$ & $0.0150(15)$ & $0.0089(14)$ \\
O3 & $0.079(3)$ & $0.0309(19)$ & $0.037(2)$ & $-0.0093(17)$ & $0.0314(19)$ & $-0.0065(14)$ \\
O4 & $0.0460(18)$ & $0.0266(17)$ & $0.0375(16)$ & $-0.0064(13)$ & $0.0171(14)$ & $-0.0077(13)$ \\
N1 & $0.0339(19)$ & $0.0234(19)$ & $0.0235(18)$ & $-0.0009(14)$ & $0.0084(14)$ & $0.0004(13)$ \\
N2 & $0.0320(19)$ & $0.027(2)$ & $0.0231(18)$ & $-0.0006(14)$ & $0.0081(15)$ & $-0.0003(13)$ \\
N3 & $0.0337(19)$ & $0.0282(19)$ & $0.0223(18)$ & $-0.0009(15)$ & $0.0077(14)$ & $0.0010(14)$ \\
N4 & $0.049(2)$ & $0.0326(19)$ & $0.0237(17)$ & $-0.0122(17)$ & $0.0103(16)$ & $-0.0023(16)$ \\
C1 & $0.029(2)$ & $0.025(2)$ & $0.023(2)$ & $0.0003(16)$ & $0.0110(17)$ & $0.0009(16)$ \\
C2 & $0.027(2)$ & $0.027(2)$ & $0.024(2)$ & $0.0006(17)$ & $0.0066(16)$ & $0.0011(16)$ \\
C3 & $0.032(2)$ & $0.023(2)$ & $0.027(2)$ & $0.0005(17)$ & $0.0108(18)$ & $-0.0016(16)$ \\
C4 & $0.0233(19)$ & $0.024(2)$ & $0.022(2)$ & $-0.0001(15)$ & $0.0082(15)$ & $0.0013(15)$ \\
C5 & $0.029(2)$ & $0.024(2)$ & $0.0201(19)$ & $-0.0017(16)$ & $0.0032(16)$ & $-0.0009(16)$ \\
C6 & $0.038(2)$ & $0.029(2)$ & $0.026(2)$ & $0.0039(19)$ & $0.0090(18)$ & $-0.0011(18)$ \\
C7 & $0.040(2)$ & $0.027(2)$ & $0.036(2)$ & $0.0047(19)$ & $0.006(2)$ & $-0.0035(19)$ \\
C8 & $0.043(3)$ & $0.022(2)$ & $0.034(2)$ & $0.0019(19)$ & $0.0007(19)$ & $0.0013(18)$ \\
C9 & $0.038(2)$ & $0.029(2)$ & $0.024(2)$ & $-0.0046(18)$ & $0.0027(18)$ & $0.0040(18)$ \\
C10 & $0.031(2)$ & $0.025(2)$ & $0.020(2)$ & $0.0015(17)$ & $0.0040(16)$ & $0.0015(16)$ \\
C11 & $0.042(3)$ & $0.034(2)$ & $0.026(2)$ & $-0.0032(18)$ & $0.0130(18)$ & $-0.0015(18)$ \\
C12 & $0.053(3)$ & $0.039(3)$ & $0.026(2)$ & $0.001(2)$ & $0.013(2)$ & $-0.007(2)$ \\
C13 & $0.059(3)$ & $0.032(3)$ & $0.030(2)$ & $-0.003(2)$ & $0.003(2)$ & $-0.0083(19)$ \\
C14 & $0.066(3)$ & $0.031(2)$ & $0.035(3)$ & $-0.018(2)$ & $0.012(2)$ & $0.002(2)$ \\
C15 & $0.038(2)$ & $0.030(2)$ & $0.023(2)$ & $-0.002(2)$ & $0.0008(17)$ & $0.0001(17)$ \\
C16 & $0.037(2)$ & $0.022(2)$ & $0.025(2)$ & $0.0026(17)$ & $0.0038(17)$ & $-0.0003(17)$ \\
C17 & $0.069(4)$ & $0.039(3)$ & $0.045(3)$ & $-0.012(3)$ & $0.005(3)$ & $0.017(2)$ \\
C18 & $0.047(3)$ & $0.024(2)$ & $0.046(3)$ & $-0.0043(19)$ & $0.013(2)$ & $-0.006(2)$ \\
& & & & & & \\
\hline
\end{tabular}

Geometric parameters $\left(A,{ }^{\circ}\right)$

\begin{tabular}{llll}
\hline $\mathrm{O} 1-\mathrm{C} 15$ & $1.202(6)$ & $\mathrm{C} 6-\mathrm{C} 7$ & $1.387(6)$ \\
$\mathrm{O} 2-\mathrm{C} 15$ & $1.324(6)$ & $\mathrm{C} 6-\mathrm{H} 6$ & 0.9300 \\
$\mathrm{O} 2-\mathrm{C} 17$ & $1.455(5)$ & $\mathrm{C} 7-\mathrm{C} 8$ & $1.373(7)$ \\
$\mathrm{O} 3-\mathrm{C} 16$ & $1.200(6)$ & $\mathrm{C} 7-\mathrm{H} 7$ & 0.9300 \\
$\mathrm{O} 4-\mathrm{C} 16$ & $1.325(6)$ & $\mathrm{C} 8-\mathrm{C} 9$ & $1.387(7)$ \\
$\mathrm{O} 4-\mathrm{C} 18$ & $1.446(5)$ & $\mathrm{C} 8-\mathrm{H} 8$ & 0.9300 \\
$\mathrm{~N} 1-\mathrm{C} 1$ & $1.329(5)$ & $\mathrm{C} 9-\mathrm{H} 9$ & 0.9300 \\
$\mathrm{~N} 1-\mathrm{C} 2$ & $1.340(5)$ & $\mathrm{C} 10-\mathrm{C} 11$ & $1.388(6)$ \\
$\mathrm{N} 2-\mathrm{C} 3$ & $1.331(6)$ & $\mathrm{C} 11-\mathrm{C} 12$ & $1.377(7)$ \\
$\mathrm{N} 2-\mathrm{C} 4$ & $1.341(5)$ & $\mathrm{C} 11-\mathrm{H} 11$ & 0.9300
\end{tabular}




\begin{tabular}{|c|c|c|c|}
\hline $\mathrm{N} 3-\mathrm{C} 9$ & $1.340(5)$ & $\mathrm{C} 12-\mathrm{C} 13$ & $1.368(7)$ \\
\hline $\mathrm{N} 3-\mathrm{C} 5$ & $1.343(5)$ & $\mathrm{C} 12-\mathrm{H} 12$ & 0.9300 \\
\hline $\mathrm{N} 4-\mathrm{C} 10$ & $1.335(6)$ & $\mathrm{C} 13-\mathrm{C} 14$ & $1.389(7)$ \\
\hline $\mathrm{N} 4-\mathrm{C} 14$ & $1.336(6)$ & $\mathrm{C} 13-\mathrm{H} 13$ & 0.9300 \\
\hline $\mathrm{C} 1-\mathrm{C} 4$ & $1.419(5)$ & $\mathrm{C} 14-\mathrm{H} 14$ & 0.9300 \\
\hline $\mathrm{C} 1-\mathrm{C} 10$ & $1.489(6)$ & C17-H17A & 0.9600 \\
\hline $\mathrm{C} 2-\mathrm{C} 3$ & $1.397(5)$ & C17-H17B & 0.9600 \\
\hline $\mathrm{C} 2-\mathrm{C} 15$ & $1.505(6)$ & $\mathrm{C} 17-\mathrm{H} 17 \mathrm{C}$ & 0.9600 \\
\hline $\mathrm{C} 3-\mathrm{C} 16$ & $1.508(6)$ & $\mathrm{C} 18-\mathrm{H} 18 \mathrm{~A}$ & 0.9600 \\
\hline $\mathrm{C} 4-\mathrm{C} 5$ & $1.495(6)$ & $\mathrm{C} 18-\mathrm{H} 18 \mathrm{~B}$ & 0.9600 \\
\hline $\mathrm{C} 5-\mathrm{C} 6$ & $1.377(6)$ & $\mathrm{C} 18-\mathrm{H} 18 \mathrm{C}$ & 0.9600 \\
\hline $\mathrm{C} 15-\mathrm{O} 2-\mathrm{C} 17$ & $115.7(4)$ & $\mathrm{N} 4-\mathrm{C} 10-\mathrm{C} 11$ & $123.2(4)$ \\
\hline $\mathrm{C} 16-\mathrm{O} 4-\mathrm{C} 18$ & $116.2(3)$ & $\mathrm{N} 4-\mathrm{C} 10-\mathrm{C} 1$ & $117.0(3)$ \\
\hline $\mathrm{C} 1-\mathrm{N} 1-\mathrm{C} 2$ & $117.5(3)$ & $\mathrm{C} 11-\mathrm{C} 10-\mathrm{C} 1$ & $119.7(4)$ \\
\hline $\mathrm{C} 3-\mathrm{N} 2-\mathrm{C} 4$ & $116.6(3)$ & $\mathrm{C} 12-\mathrm{C} 11-\mathrm{C} 10$ & $119.1(4)$ \\
\hline $\mathrm{C} 9-\mathrm{N} 3-\mathrm{C} 5$ & $116.7(4)$ & $\mathrm{C} 12-\mathrm{C} 11-\mathrm{H} 11$ & 120.5 \\
\hline $\mathrm{C} 10-\mathrm{N} 4-\mathrm{C} 14$ & $116.5(4)$ & $\mathrm{C} 10-\mathrm{C} 11-\mathrm{H} 11$ & 120.5 \\
\hline $\mathrm{N} 1-\mathrm{C} 1-\mathrm{C} 4$ & $121.1(4)$ & $\mathrm{C} 13-\mathrm{C} 12-\mathrm{C} 11$ & $118.8(4)$ \\
\hline $\mathrm{N} 1-\mathrm{C} 1-\mathrm{C} 10$ & $116.2(3)$ & $\mathrm{C} 13-\mathrm{C} 12-\mathrm{H} 12$ & 120.6 \\
\hline $\mathrm{C} 4-\mathrm{C} 1-\mathrm{C} 10$ & $122.7(4)$ & $\mathrm{C} 11-\mathrm{C} 12-\mathrm{H} 12$ & 120.6 \\
\hline $\mathrm{N} 1-\mathrm{C} 2-\mathrm{C} 3$ & $120.9(4)$ & $\mathrm{C} 12-\mathrm{C} 13-\mathrm{C} 14$ & $118.3(4)$ \\
\hline $\mathrm{N} 1-\mathrm{C} 2-\mathrm{C} 15$ & $118.3(3)$ & $\mathrm{C} 12-\mathrm{C} 13-\mathrm{H} 13$ & 120.9 \\
\hline $\mathrm{C} 3-\mathrm{C} 2-\mathrm{C} 15$ & $120.8(4)$ & $\mathrm{C} 14-\mathrm{C} 13-\mathrm{H} 13$ & 120.9 \\
\hline $\mathrm{N} 2-\mathrm{C} 3-\mathrm{C} 2$ & $122.5(4)$ & $\mathrm{N} 4-\mathrm{C} 14-\mathrm{C} 13$ & $124.2(4)$ \\
\hline $\mathrm{N} 2-\mathrm{C} 3-\mathrm{C} 16$ & $116.7(3)$ & $\mathrm{N} 4-\mathrm{C} 14-\mathrm{H} 14$ & 117.9 \\
\hline $\mathrm{C} 2-\mathrm{C} 3-\mathrm{C} 16$ & $120.8(4)$ & $\mathrm{C} 13-\mathrm{C} 14-\mathrm{H} 14$ & 117.9 \\
\hline $\mathrm{N} 2-\mathrm{C} 4-\mathrm{C} 1$ & $121.1(4)$ & $\mathrm{O} 1-\mathrm{C} 15-\mathrm{O} 2$ & $125.6(4)$ \\
\hline $\mathrm{N} 2-\mathrm{C} 4-\mathrm{C} 5$ & $115.8(3)$ & $\mathrm{O} 1-\mathrm{C} 15-\mathrm{C} 2$ & $122.9(4)$ \\
\hline $\mathrm{C} 1-\mathrm{C} 4-\mathrm{C} 5$ & $122.8(4)$ & $\mathrm{O} 2-\mathrm{C} 15-\mathrm{C} 2$ & $111.6(4)$ \\
\hline $\mathrm{N} 3-\mathrm{C} 5-\mathrm{C} 6$ & $123.2(4)$ & $\mathrm{O} 3-\mathrm{C} 16-\mathrm{O} 4$ & $126.2(4)$ \\
\hline $\mathrm{N} 3-\mathrm{C} 5-\mathrm{C} 4$ & $117.7(3)$ & $\mathrm{O} 3-\mathrm{C} 16-\mathrm{C} 3$ & $124.5(4)$ \\
\hline $\mathrm{C} 6-\mathrm{C} 5-\mathrm{C} 4$ & $119.1(4)$ & $\mathrm{O} 4-\mathrm{C} 16-\mathrm{C} 3$ & $109.4(3)$ \\
\hline $\mathrm{C} 5-\mathrm{C} 6-\mathrm{C} 7$ & $119.0(4)$ & $\mathrm{O} 2-\mathrm{C} 17-\mathrm{H} 17 \mathrm{~A}$ & 109.5 \\
\hline $\mathrm{C} 5-\mathrm{C} 6-\mathrm{H} 6$ & 120.5 & $\mathrm{O} 2-\mathrm{C} 17-\mathrm{H} 17 \mathrm{~B}$ & 109.5 \\
\hline $\mathrm{C} 7-\mathrm{C} 6-\mathrm{H} 6$ & 120.5 & H17A-C17-H17B & 109.5 \\
\hline $\mathrm{C} 8-\mathrm{C} 7-\mathrm{C} 6$ & $118.9(4)$ & $\mathrm{O} 2-\mathrm{C} 17-\mathrm{H} 17 \mathrm{C}$ & 109.5 \\
\hline $\mathrm{C} 8-\mathrm{C} 7-\mathrm{H} 7$ & 120.5 & $\mathrm{H} 17 \mathrm{~A}-\mathrm{C} 17-\mathrm{H} 17 \mathrm{C}$ & 109.5 \\
\hline $\mathrm{C} 6-\mathrm{C} 7-\mathrm{H} 7$ & 120.5 & $\mathrm{H} 17 \mathrm{~B}-\mathrm{C} 17-\mathrm{H} 17 \mathrm{C}$ & 109.5 \\
\hline $\mathrm{C} 7-\mathrm{C} 8-\mathrm{C} 9$ & $118.3(4)$ & $\mathrm{O} 4-\mathrm{C} 18-\mathrm{H} 18 \mathrm{~A}$ & 109.5 \\
\hline $\mathrm{C} 7-\mathrm{C} 8-\mathrm{H} 8$ & 120.9 & $\mathrm{O} 4-\mathrm{C} 18-\mathrm{H} 18 \mathrm{~B}$ & 109.5 \\
\hline $\mathrm{C} 9-\mathrm{C} 8-\mathrm{H} 8$ & 120.9 & $\mathrm{H} 18 \mathrm{~A}-\mathrm{C} 18-\mathrm{H} 18 \mathrm{~B}$ & 109.5 \\
\hline $\mathrm{N} 3-\mathrm{C} 9-\mathrm{C} 8$ & $123.9(4)$ & $\mathrm{O} 4-\mathrm{C} 18-\mathrm{H} 18 \mathrm{C}$ & 109.5 \\
\hline $\mathrm{N} 3-\mathrm{C} 9-\mathrm{H} 9$ & 118.1 & $\mathrm{H} 18 \mathrm{~A}-\mathrm{C} 18-\mathrm{H} 18 \mathrm{C}$ & 109.5 \\
\hline $\mathrm{C} 8-\mathrm{C} 9-\mathrm{H} 9$ & 118.1 & $\mathrm{H} 18 \mathrm{~B}-\mathrm{C} 18-\mathrm{H} 18 \mathrm{C}$ & 109.5 \\
\hline $\mathrm{C} 2-\mathrm{N} 1-\mathrm{C} 1-\mathrm{C} 4$ & $-3.6(5)$ & $\mathrm{C} 5-\mathrm{N} 3-\mathrm{C} 9-\mathrm{C} 8$ & $0.1(6)$ \\
\hline $\mathrm{C} 2-\mathrm{N} 1-\mathrm{C} 1-\mathrm{C} 10$ & $175.4(3)$ & $\mathrm{C} 7-\mathrm{C} 8-\mathrm{C} 9-\mathrm{N} 3$ & $0.4(7)$ \\
\hline
\end{tabular}




$\begin{array}{llll}\mathrm{C} 1-\mathrm{N} 1-\mathrm{C} 2-\mathrm{C} 3 & -1.6(5) & \mathrm{C} 14-\mathrm{N} 4-\mathrm{C} 10-\mathrm{C} 11 & -0.4(7) \\ \mathrm{C} 1-\mathrm{N} 1-\mathrm{C} 2-\mathrm{C} 15 & -178.2(4) & \mathrm{C} 14-\mathrm{N} 4-\mathrm{C} 10-\mathrm{C} 1 & 176.0(4) \\ \mathrm{C} 4-\mathrm{N} 2-\mathrm{C} 3-\mathrm{C} 2 & -1.4(5) & \mathrm{N} 1-\mathrm{C} 1-\mathrm{C} 10-\mathrm{N} 4 & -133.7(4) \\ \mathrm{C} 4-\mathrm{N} 2-\mathrm{C} 3-\mathrm{C} 16 & -178.6(4) & \mathrm{C} 4-\mathrm{C} 1-\mathrm{C} 10-\mathrm{N} 4 & 45.3(5) \\ \mathrm{N} 1-\mathrm{C} 2-\mathrm{C} 3-\mathrm{N} 2 & 4.3(6) & \mathrm{N} 1-\mathrm{C} 1-\mathrm{C} 10-\mathrm{C} 11 & 42.8(5) \\ \mathrm{C} 15-\mathrm{C} 2-\mathrm{C} 3-\mathrm{N} 2 & -179.2(4) & \mathrm{C} 4-\mathrm{C} 1-\mathrm{C} 10-\mathrm{C} 11 & -138.2(4) \\ \mathrm{N} 1-\mathrm{C} 2-\mathrm{C} 3-\mathrm{C} 16 & -178.6(4) & \mathrm{N} 4-\mathrm{C} 10-\mathrm{C} 11-\mathrm{C} 12 & -0.7(7) \\ \mathrm{C} 15-\mathrm{C} 2-\mathrm{C} 3-\mathrm{C} 16 & -2.1(6) & \mathrm{C} 1-\mathrm{C} 10-\mathrm{C} 11-\mathrm{C} 12 & -177.0(4) \\ \mathrm{C} 3-\mathrm{N} 2-\mathrm{C} 4-\mathrm{C} 1 & -3.8(5) & \mathrm{C} 10-\mathrm{C} 11-\mathrm{C} 12-\mathrm{C} 13 & 1.2(7) \\ \mathrm{C} 3-\mathrm{N} 2-\mathrm{C} 4-\mathrm{C} 5 & 170.5(3) & \mathrm{C} 11-\mathrm{C} 12-\mathrm{C} 13-\mathrm{C} 14 & -0.6(8) \\ \mathrm{N} 1-\mathrm{C} 1-\mathrm{C} 4-\mathrm{N} 2 & 6.5(5) & \mathrm{C} 10-\mathrm{N} 4-\mathrm{C} 14-\mathrm{C} 13 & 1.0(8) \\ \mathrm{C} 10-\mathrm{C} 1-\mathrm{C} 4-\mathrm{N} 2 & -172.4(4) & \mathrm{C} 12-\mathrm{C} 13-\mathrm{C} 14-\mathrm{N} 4 & -0.5(8) \\ \mathrm{N} 1-\mathrm{C} 1-\mathrm{C} 4-\mathrm{C} 5 & -167.4(4) & \mathrm{C} 17-\mathrm{O} 2-\mathrm{C} 15-\mathrm{O} 1 & 4.4(7) \\ \mathrm{C} 10-\mathrm{C} 1-\mathrm{C} 4-\mathrm{C} 5 & 13.7(5) & \mathrm{C} 17-\mathrm{O} 2-\mathrm{C} 15-\mathrm{C} 2 & -174.1(4) \\ \mathrm{C} 9-\mathrm{N} 3-\mathrm{C} 5-\mathrm{C} 6 & -0.6(6) & \mathrm{N} 1-\mathrm{C} 2-\mathrm{C} 15-\mathrm{O} 1 & 120.0(5) \\ \mathrm{C} 9-\mathrm{N} 3-\mathrm{C} 5-\mathrm{C} 4 & -177.9(4) & \mathrm{C} 3-\mathrm{C} 2-\mathrm{C} 15-\mathrm{O} 1 & -56.6(6) \\ \mathrm{N} 2-\mathrm{C} 4-\mathrm{C} 5-\mathrm{N} 3 & 50.7(5) & \mathrm{N} 1-\mathrm{C} 2-\mathrm{C} 15-\mathrm{O} 2 & -61.5(5) \\ \mathrm{C} 1-\mathrm{C} 4-\mathrm{C} 5-\mathrm{N} 3 & -135.1(4) & \mathrm{C} 3-\mathrm{C} 2-\mathrm{C} 15-\mathrm{O} 2 & 121.9(4) \\ \mathrm{N} 2-\mathrm{C} 4-\mathrm{C} 5-\mathrm{C} 6 & -126.6(4) & \mathrm{C} 18-\mathrm{O} 4-\mathrm{C} 16-\mathrm{O} 3 & -6.2(7) \\ \mathrm{C} 1-\mathrm{C} 4-\mathrm{C} 5-\mathrm{C} 6 & 47.6(6) & \mathrm{C} 18-\mathrm{O} 4-\mathrm{C} 16-\mathrm{C} 3 & 173.9(4) \\ \mathrm{N} 3-\mathrm{C} 5-\mathrm{C} 6-\mathrm{C} 7 & 0.7(7) & \mathrm{N} 2-\mathrm{C} 3-\mathrm{C} 16-\mathrm{O} 3 & -50.9(6) \\ \mathrm{C} 4-\mathrm{C} 5-\mathrm{C} 6-\mathrm{C} 7 & 177.9(4) & \mathrm{C} 2-\mathrm{C} 3-\mathrm{C} 16-\mathrm{O} 3 & 131.8(5) \\ \mathrm{C} 5-\mathrm{C} 6-\mathrm{C} 7-\mathrm{C} 8 & -0.2(7) & \mathrm{N} 2-\mathrm{C} 3-\mathrm{C} 16-\mathrm{O} 4 & 128.9(4) \\ \mathrm{C} 6-\mathrm{C} 7-\mathrm{C} 8-\mathrm{C} 9 & -0.4(7) & \mathrm{C} 2-\mathrm{C} 3-\mathrm{C} 16-\mathrm{O} 4 & -48.3(5) \\ & & & \end{array}$

Hydrogen-bond geometry $\left(\AA,{ }^{\circ}\right)$

$\mathrm{Cg} 2$ is the centroid of the $\mathrm{N} 3 / \mathrm{C} 5-\mathrm{C} 9$ pyridine ring.

\begin{tabular}{lllll}
\hline$D-\mathrm{H} \cdots A$ & $D-\mathrm{H}$ & $\mathrm{H} \cdots A$ & $D \cdots A$ & $D-\mathrm{H} \cdots A$ \\
\hline $\mathrm{C} 11-\mathrm{H} 11 \cdots \mathrm{N} 3^{\mathrm{i}}$ & 0.93 & 2.57 & $3.334(5)$ & 140 \\
$\mathrm{C} 7-\mathrm{H} 7 \cdots C g 2^{\mathrm{ii}}$ & 0.93 & 2.95 & $3.742(5)$ & 144 \\
$\mathrm{C} 17-\mathrm{H} 17 C \cdots C g 2^{\mathrm{iii}}$ & 0.96 & 2.92 & $3.722(6)$ & 141 \\
\hline
\end{tabular}

Symmetry codes: (i) $x,-y+3 / 2, z-1 / 2$; (ii) $x+1 / 2,-y+1$, $z$; (iii) $x-1 / 2, y+3 / 2, z-1 / 2$.

(II) Diethyl 5,6-bis(pyridin-2-yl)pyrazine-2,3-dicarboxylate

Crystal data

$\mathrm{C}_{20} \mathrm{H}_{18} \mathrm{~N}_{4} \mathrm{O}_{4}$

$M_{r}=378.38$

Tetragonal, $I 4_{1} / a$

$a=10.2295(6) \AA$

$c=36.281(3) \AA$

$V=3796.5(5) \AA^{3}$

$Z=8$

$F(000)=1584$
$D_{\mathrm{x}}=1.324 \mathrm{Mg} \mathrm{m}^{-3}$

Mo $K \alpha$ radiation, $\lambda=0.71073 \AA$

Cell parameters from 5000 reflections

$\theta=3.3-52.1^{\circ}$

$\mu=0.10 \mathrm{~mm}^{-1}$

$T=223 \mathrm{~K}$

Block, colourless

$0.65 \times 0.50 \times 0.50 \mathrm{~mm}$ 


\section{Data collection}

Stoe IPDS 1

diffractometer

Radiation source: fine-focus sealed tube

Plane graphite monochromator

$\varphi$ rotation scans

14760 measured reflections

1851 independent reflections

\section{Refinement}

Refinement on $F^{2}$

Least-squares matrix: full

$R\left[F^{2}>2 \sigma\left(F^{2}\right)\right]=0.049$

$w R\left(F^{2}\right)=0.149$

$S=1.01$

1851 reflections

129 parameters

0 restraints

Primary atom site location: structure-invariant direct methods

Secondary atom site location: difference Fourier map

\section{Special details}

Geometry. All e.s.d.'s (except the e.s.d. in the dihedral angle between two 1.s. planes) are estimated using the full covariance matrix. The cell e.s.d.'s are taken into account individually in the estimation of e.s.d.'s in distances, angles and torsion angles; correlations between e.s.d.'s in cell parameters are only used when they are defined by crystal symmetry. An approximate (isotropic) treatment of cell e.s.d.'s is used for estimating e.s.d.'s involving 1.s. planes.

Fractional atomic coordinates and isotropic or equivalent isotropic displacement parameters $\left(\AA^{2}\right)$

\begin{tabular}{lllll}
\hline & $x$ & $y$ & $z$ & $U_{\text {iso }} * / U_{\text {eq }}$ \\
\hline O1 & $0.7424(2)$ & $0.7559(3)$ & $0.00480(5)$ & $0.1009(9)$ \\
O2 & $0.91015(17)$ & $0.64422(18)$ & $-0.01674(4)$ & $0.0693(5)$ \\
N1 & $0.86590(16)$ & $0.73125(18)$ & $0.07318(4)$ & $0.0478(5)$ \\
N2 & $0.88149(17)$ & $0.8399(2)$ & $0.16340(5)$ & $0.0583(6)$ \\
C1 & $0.9332(2)$ & $0.7371(2)$ & $0.04156(5)$ & $0.0473(5)$ \\
C2 & $0.93113(19)$ & $0.7446(2)$ & $0.10488(5)$ & $0.0450(5)$ \\
C3 & $0.84753(19)$ & $0.7504(2)$ & $0.13824(5)$ & $0.0452(5)$ \\
C4 & $0.7388(2)$ & $0.6719(2)$ & $0.14134(5)$ & $0.0466(5)$ \\
H4 & 0.7174 & 0.6124 & 0.1225 & $0.056^{*}$ \\
C5 & $0.6613(2)$ & $0.6817(2)$ & $0.17249(5)$ & $0.0503(5)$ \\
H5 & 0.5874 & 0.6282 & 0.1755 & $0.060^{*}$ \\
C6 & $0.6952(2)$ & $0.7712(2)$ & $0.19868(6)$ & $0.0569(6)$ \\
H6 & 0.6448 & 0.7803 & 0.2202 & $0.068^{*}$ \\
C7 & $0.8042(2)$ & $0.8481(3)$ & $0.19322(6)$ & $0.0637(7)$ \\
H7 & 0.8256 & 0.9098 & 0.2114 & $0.076^{*}$ \\
C8 & $0.8508(2)$ & $0.7155(3)$ & $0.00796(5)$ & $0.0558(6)$ \\
C9 & $0.8402(3)$ & $0.6183(4)$ & $-0.05106(7)$ & $0.0978(11)$ \\
H9A & 0.7744 & 0.5501 & -0.0471 & $0.117^{*}$ \\
H9B & 0.7956 & 0.6977 & -0.0594 & $0.117^{*}$
\end{tabular}

1153 reflections with $I>2 \sigma(I)$

$R_{\text {int }}=0.043$

$\theta_{\max }=26.0^{\circ}, \theta_{\min }=2.1^{\circ}$

$h=-12 \rightarrow 12$

$k=-12 \rightarrow 12$

$l=-44 \rightarrow 44$

Hydrogen site location: inferred from neighbouring sites

$\mathrm{H}$-atom parameters constrained

$w=1 /\left[\sigma^{2}\left(F_{\mathrm{o}}{ }^{2}\right)+(0.0925 P)^{2}\right]$

where $P=\left(F_{\mathrm{o}}^{2}+2 F_{\mathrm{c}}^{2}\right) / 3$

$(\Delta / \sigma)_{\max }<0.001$

$\Delta \rho_{\max }=0.34 \mathrm{e} \AA^{-3}$

$\Delta \rho_{\min }=-0.19$ e $\AA^{-3}$

Extinction correction: SHELXL2014 (Sheldrick, 2015), $\mathrm{Fc}^{*}=\mathrm{kFc}\left[1+0.001 \mathrm{xFc}^{2} \lambda^{3} / \sin (2 \theta)\right]^{-1 / 4}$

Extinction coefficient: 0.0049 (10) 


\begin{tabular}{lllll} 
C10 & $0.9335(4)$ & $0.5760(3)$ & $-0.07867(8)$ & $0.0965(11)$ \\
H10A & 0.8877 & 0.5552 & -0.1013 & $0.145^{*}$ \\
H10B & 0.9793 & 0.4990 & -0.0699 & $0.145^{*}$ \\
H10C & 0.9960 & 0.6455 & -0.0833 & $0.145^{*}$ \\
\hline
\end{tabular}

Atomic displacement parameters $\left(\AA^{2}\right)$

\begin{tabular}{lllllll}
\hline & $U^{11}$ & $U^{22}$ & $U^{33}$ & $U^{12}$ & $U^{13}$ & $U^{23}$ \\
\hline O1 & $0.0662(13)$ & $0.187(2)$ & $0.0499(11)$ & $0.0406(14)$ & $-0.0132(9)$ & $-0.0026(12)$ \\
O2 & $0.0689(11)$ & $0.0889(12)$ & $0.0500(9)$ & $0.0023(9)$ & $-0.0168(8)$ & $-0.0172(8)$ \\
N1 & $0.0424(9)$ & $0.0665(12)$ & $0.0347(9)$ & $0.0012(8)$ & $-0.0018(7)$ & $0.0026(8)$ \\
N2 & $0.0464(10)$ & $0.0866(14)$ & $0.0418(9)$ & $-0.0104(10)$ & $0.0042(8)$ & $-0.0093(9)$ \\
C1 & $0.0440(10)$ & $0.0630(13)$ & $0.0348(10)$ & $0.0039(10)$ & $-0.0018(8)$ & $0.0012(9)$ \\
C2 & $0.0418(10)$ & $0.0581(13)$ & $0.0350(10)$ & $-0.0016(9)$ & $-0.0010(8)$ & $0.0011(9)$ \\
C3 & $0.0379(10)$ & $0.0629(13)$ & $0.0348(10)$ & $0.0007(9)$ & $-0.0026(8)$ & $0.0026(9)$ \\
C4 & $0.0421(11)$ & $0.0541(12)$ & $0.0437(11)$ & $0.0028(9)$ & $-0.0017(8)$ & $0.0041(9)$ \\
C5 & $0.0407(11)$ & $0.0630(13)$ & $0.0471(12)$ & $0.0017(10)$ & $0.0035(9)$ & $0.0086(10)$ \\
C6 & $0.0450(12)$ & $0.0826(17)$ & $0.0431(11)$ & $0.0052(12)$ & $0.0067(9)$ & $0.0062(11)$ \\
C7 & $0.0553(14)$ & $0.0941(18)$ & $0.0416(11)$ & $-0.0080(13)$ & $0.0046(10)$ & $-0.0127(12)$ \\
C8 & $0.0467(13)$ & $0.0851(17)$ & $0.0357(11)$ & $0.0035(12)$ & $-0.0008(9)$ & $0.0047(11)$ \\
C9 & $0.095(2)$ & $0.141(3)$ & $0.0578(16)$ & $-0.007(2)$ & $-0.0290(15)$ & $-0.0268(18)$ \\
C10 & $0.157(3)$ & $0.0720(18)$ & $0.0605(17)$ & $-0.020(2)$ & $-0.0135(19)$ & $-0.0139(14)$ \\
& & & & & & \\
\hline
\end{tabular}

Geometric parameters $\left(\AA,{ }^{\circ}\right)$

\begin{tabular}{llll}
\hline $\mathrm{O} 1-\mathrm{C} 8$ & $1.189(3)$ & $\mathrm{C} 4-\mathrm{H} 4$ & 0.9400 \\
$\mathrm{O} 2-\mathrm{C} 8$ & $1.305(3)$ & $\mathrm{C} 5-\mathrm{C} 6$ & $1.364(3)$ \\
$\mathrm{O} 2-\mathrm{C} 9$ & $1.460(3)$ & $\mathrm{C} 5-\mathrm{H} 5$ & 0.9400 \\
$\mathrm{~N} 1-\mathrm{C} 2$ & $1.337(2)$ & $\mathrm{C} 6-\mathrm{C} 7$ & $1.379(3)$ \\
$\mathrm{N} 1-\mathrm{C} 1$ & $1.339(2)$ & $\mathrm{C} 6-\mathrm{H} 6$ & 0.9400 \\
$\mathrm{~N} 2-\mathrm{C} 3$ & $1.339(3)$ & $\mathrm{C} 7-\mathrm{H} 7$ & 0.9400 \\
$\mathrm{~N} 2-\mathrm{C} 7$ & $1.343(3)$ & $\mathrm{C} 9-\mathrm{C} 10$ & $1.450(5)$ \\
$\mathrm{C} 1-\mathrm{C} 1^{\mathrm{i}}$ & $1.392(4)$ & $\mathrm{C} 9-\mathrm{H} 9 \mathrm{~A}$ & 0.9800 \\
$\mathrm{C} 1-\mathrm{C} 8$ & $1.499(3)$ & $\mathrm{C} 9-\mathrm{H} 9 \mathrm{~B}$ & 0.9800 \\
$\mathrm{C} 2-\mathrm{C} 2 \mathrm{i}$ & $1.413(4)$ & $\mathrm{C} 10-\mathrm{H} 10 \mathrm{~A}$ & 0.9700 \\
$\mathrm{C} 2-\mathrm{C} 3$ & $1.483(3)$ & $\mathrm{C} 10-\mathrm{H} 10 \mathrm{~B}$ & 0.9700 \\
$\mathrm{C} 3-\mathrm{C} 4$ & $1.377(3)$ & $\mathrm{C} 10-\mathrm{H} 10 \mathrm{C}$ & 0.9700 \\
$\mathrm{C} 4-\mathrm{C} 5$ & $1.384(3)$ & & 120.4 \\
& & & $123.8(2)$ \\
$\mathrm{C} 8-\mathrm{O} 2-\mathrm{C} 9$ & $117.3(2)$ & $\mathrm{C} 7-\mathrm{C} 6-\mathrm{H} 6$ & 118.1 \\
$\mathrm{C} 2-\mathrm{N} 1-\mathrm{C} 1$ & $118.40(17)$ & $\mathrm{N} 2-\mathrm{C} 7-\mathrm{C} 6$ & 118.1 \\
$\mathrm{C} 3-\mathrm{N} 2-\mathrm{C} 7$ & $116.06(19)$ & $\mathrm{N} 2-\mathrm{C} 7-\mathrm{H} 7$ & $124.2(2)$ \\
$\mathrm{N} 1-\mathrm{C} 1-\mathrm{C} 1^{\mathrm{i}}$ & $120.88(11)$ & $\mathrm{C} 6-\mathrm{C} 7-\mathrm{H} 7$ & $123.5(2)$ \\
$\mathrm{N} 1-\mathrm{C} 1-\mathrm{C} 8$ & $113.63(18)$ & $\mathrm{O} 1-\mathrm{C} 8-\mathrm{O} 2$ & $112.27(19)$ \\
$\mathrm{C} 1-\mathrm{C} 1-\mathrm{C} 8$ & $125.48(12)$ & $\mathrm{O} 1-\mathrm{C} 8-\mathrm{C} 1$ & $108.7(3)$ \\
$\mathrm{N} 1-\mathrm{C} 2-\mathrm{C} 2^{\mathrm{i}}$ & $120.38(11)$ & $\mathrm{O} 2-\mathrm{C} 8-\mathrm{C} 1$ & 109.9 \\
$\mathrm{~N} 1-\mathrm{C} 2-\mathrm{C} 3$ & $114.72(17)$ & $\mathrm{C} 10-\mathrm{C} 9-\mathrm{O} 2$ & \\
$\mathrm{C} 2-\mathrm{C} 2-\mathrm{C} 3$ & $124.88(11)$ & $\mathrm{C} 10-\mathrm{C} 9-\mathrm{H} 9 \mathrm{~A}$ &
\end{tabular}




$\begin{array}{llll}\mathrm{N} 2-\mathrm{C} 3-\mathrm{C} 4 & 123.56(18) & \mathrm{O} 2-\mathrm{C} 9-\mathrm{H} 9 \mathrm{~A} & 109.9 \\ \mathrm{~N} 2-\mathrm{C} 3-\mathrm{C} 2 & 115.71(18) & \mathrm{C} 10-\mathrm{C} 9-\mathrm{H} 9 \mathrm{~B} & 109.9 \\ \mathrm{C} 4-\mathrm{C} 3-\mathrm{C} 2 & 120.65(18) & \mathrm{O} 2-\mathrm{C} 9-\mathrm{H} 9 \mathrm{~B} & 109.9 \\ \mathrm{C} 3-\mathrm{C} 4-\mathrm{C} 5 & 119.1(2) & \mathrm{H} 9 \mathrm{~A}-\mathrm{C} 9-\mathrm{H} 9 \mathrm{~B} & 108.3 \\ \mathrm{C} 3-\mathrm{C} 4-\mathrm{H} 4 & 120.4 & \mathrm{C} 9-\mathrm{C} 10-\mathrm{H} 10 \mathrm{~A} & 109.5 \\ \mathrm{C} 5-\mathrm{C} 4-\mathrm{H} 4 & 120.4 & \mathrm{C} 9-\mathrm{C} 10-\mathrm{H} 10 \mathrm{~B} & 109.5 \\ \mathrm{C} 6-\mathrm{C} 5-\mathrm{C} 4 & 118.2(2) & \mathrm{H} 10 \mathrm{~A}-\mathrm{C} 10-\mathrm{H} 10 \mathrm{~B} & 109.5 \\ \mathrm{C} 6-\mathrm{C} 5-\mathrm{H} 5 & 120.9 & \mathrm{C} 9-\mathrm{C} 10-\mathrm{H} 10 \mathrm{C} & 109.5 \\ \mathrm{C} 4-\mathrm{C} 5-\mathrm{H} 5 & 120.9 & \mathrm{H} 10 \mathrm{~A}-\mathrm{C} 10-\mathrm{H} 10 \mathrm{C} & 109.5 \\ \mathrm{C} 5-\mathrm{C} 6-\mathrm{C} 7 & 119.20(19) & \mathrm{H} 10 \mathrm{~B}-\mathrm{C} 10-\mathrm{H} 10 \mathrm{C} & \\ \mathrm{C} 5-\mathrm{C} 6-\mathrm{H} 6 & 120.4 & & 1.1(3) \\ & & \mathrm{C} 3-\mathrm{C} 4-\mathrm{C} 5-\mathrm{C} 6 & 0.1(3) \\ \mathrm{C} 2-\mathrm{N} 1-\mathrm{C} 1-\mathrm{C} 1{ }^{\mathrm{i}} & -3.1(4) & \mathrm{C} 4-\mathrm{C} 5-\mathrm{C} 6-\mathrm{C} 7 & -0.2(4) \\ \mathrm{C} 2-\mathrm{N} 1-\mathrm{C} 1-\mathrm{C} 8 & 177.7(2) & \mathrm{C} 3-\mathrm{N} 2-\mathrm{C} 7-\mathrm{C} 6 & -0.6(4) \\ \mathrm{C} 1-\mathrm{N} 1-\mathrm{C} 2-\mathrm{C} 2 \mathrm{i} & -4.4(4) & \mathrm{C} 5-\mathrm{C} 6-\mathrm{C} 7-\mathrm{N} 2 & 3.0(4) \\ \mathrm{C} 1-\mathrm{N} 1-\mathrm{C} 2-\mathrm{C} 3 & 174.09(19) & \mathrm{C} 9-\mathrm{O} 2-\mathrm{C} 8-\mathrm{O} 1 & -179.0(2) \\ \mathrm{C} 7-\mathrm{N} 2-\mathrm{C} 3-\mathrm{C} 4 & 1.6(3) & \mathrm{C} 9-\mathrm{O} 2-\mathrm{C} 8-\mathrm{C} 1 & 37.9(4) \\ \mathrm{C} 7-\mathrm{N} 2-\mathrm{C} 3-\mathrm{C} 2 & 178.3(2) & \mathrm{C} 1-\mathrm{C} 1-\mathrm{C} 8-\mathrm{O} 1 & -141.2(3) \\ \mathrm{N} 1-\mathrm{C} 2-\mathrm{C} 3-\mathrm{N} 2 & -137.7(2) & \mathrm{N} 1-\mathrm{C} 1-\mathrm{C} 8-\mathrm{O} 1 & -140.1(2) \\ \mathrm{C} 2-\mathrm{C} 2-\mathrm{C} 3-\mathrm{N} 2 & 40.8(4) & \mathrm{C} 1-\mathrm{C} 1-\mathrm{C} 8-\mathrm{O} 2 & 40.8(4) \\ \mathrm{N} 1-\mathrm{C} 2-\mathrm{C} 3-\mathrm{C} 4 & 39.1(3) & \mathrm{C} 8-\mathrm{O} 2-\mathrm{C} 9-\mathrm{C} 10 & 162.0(3) \\ \mathrm{C} 2-\mathrm{C} 2-\mathrm{C} 3-\mathrm{C} 4 & -142.4(3) & -2.1(3) & \end{array}$

Symmetry code: (i) $-x+2,-y+3 / 2, z$.

Hydrogen-bond geometry $\left(A,{ }^{\circ}\right)$

$\mathrm{Cg} 1$ and $\mathrm{Cg} 2$ are the centroids of the pyrazine and pyridine rings $\mathrm{N} 1 / \mathrm{C} 1 / \mathrm{C} 2 / \mathrm{N} 1^{\prime} / \mathrm{C} 1^{\prime} / \mathrm{C} 2^{\prime}$ and $\mathrm{N} 2 / \mathrm{C} 3-\mathrm{C} 7$, respectively [symmetry code $\left({ }^{\prime}\right):-x+2,-y+3 / 2$, $z]$.

\begin{tabular}{lllll}
\hline$D-\mathrm{H} \cdots A$ & $D-\mathrm{H}$ & $\mathrm{H} \cdots A$ & $D \cdots A$ & $D-\mathrm{H} \cdots A$ \\
\hline $\mathrm{C} 7-\mathrm{H} 7 \cdots \mathrm{O} 1^{\mathrm{ii}}$ & 0.94 & 2.48 & $3.308(3)$ & 147 \\
$\mathrm{C} 4-\mathrm{H} 4 \cdots C g 2^{\mathrm{iii}}$ & 0.94 & 2.92 & $3.739(2)$ & 147 \\
$\mathrm{C} 10-\mathrm{H} 10 B \cdots C g 1^{\mathrm{iv}}$ & 0.97 & 2.56 & $3.409(3)$ & 146 \\
$\mathrm{C} 10-\mathrm{H} 10 B \cdots C g 1^{\mathrm{v}}$ & 0.97 & 2.56 & $3.409(3)$ & 146
\end{tabular}

Symmetry codes: (ii) $-y+7 / 4, x+1 / 4, z+1 / 4$; (iii) $y-1 / 4,-x+5 / 4,-z+1 / 4$; (iv) $-x+2,-y+1,-z$; (v) $x, y-1 / 2,-z$. 